\title{
Diversity and Evolutionary Histories of Human Coronaviruses NL63 and 229E Associated with Acute Upper Respiratory Tract Symptoms in Kuala Lumpur, Malaysia
}

\author{
Maryam Nabiel Al-Khannaq, Kim Tien Ng, Xiang Yong Oong, Yong Kek Pang, Yutaka Takebe, Jack Bee Chook, \\ Nik Sherina Hanafi, Adeeba Kamarulzaman, and Kok Keng Tee* \\ Department of Medicine, Faculty of Medicine, University of Malaya, Kuala Lumpur, Malaysia; AIDS Research Center, National Institute of \\ Infectious Diseases, Tokyo, Japan; School of Medicine, Yokohama City University, Kanagawa, Japan; Department of Health Sciences, \\ Faculty of Health and Life Sciences, Management and Science University, Selangor, Malaysia; Department of Primary Care Medicine, \\ Faculty of Medicine, University of Malaya, Kuala Lumpur, Malaysia; Department of Medical Microbiology, Faculty of Medicine, \\ University of Malaya, Kuala Lumpur, Malaysia
}

\begin{abstract}
The human alphacoronaviruses HCoV-NL63 and HCoV-229E are commonly associated with upper respiratory tract infections (URTI). Information on their molecular epidemiology and evolutionary dynamics in the tropical region of southeast Asia however is limited. Here, we analyzed the phylogenetic, temporal distribution, population history, and clinical manifestations among patients infected with HCoV-NL63 and HCoV-229E. Nasopharyngeal swabs were collected from 2,060 consenting adults presented with acute URTI symptoms in Kuala Lumpur, Malaysia, between 2012 and 2013. The presence of HCoV-NL63 and HCoV-229E was detected using multiplex polymerase chain reaction (PCR). The spike glycoprotein, nucleocapsid, and $1 a$ genes were sequenced for phylogenetic reconstruction and Bayesian coalescent inference. A total of 68/2,060 (3.3\%) subjects were positive for human alphacoronavirus; HCoV-NL63 and HCoV-229E were detected in $45(2.2 \%)$ and $23(1.1 \%)$ patients, respectively. A peak in the number of HCoV-NL63 infections was recorded between June and October 2012. Phylogenetic inference revealed that $62.8 \%$ of HCoV-NL63 infections belonged to genotype B, 37.2\% was genotype C, while all HCoV-229E sequences were clustered within group 4. Molecular dating analysis indicated that the origin of HCoV-NL63 was dated to 1921, before it diverged into genotype A (1975), genotype B (1996), and genotype C (2003). The root of the HCoV-229E tree was dated to 1955, before it diverged into groups 1-4 between the 1970s and 1990s. The study described the seasonality, molecular diversity, and evolutionary dynamics of human alphacoronavirus infections in a tropical region.
\end{abstract}

\section{INTRODUCTION}

Human coronaviruses were first reported in the mid-1960s and are known to be associated with acute upper respiratory tract infections (URTI) or the common cold. ${ }^{1-3}$ According to the International Committee for Taxonomy of Viruses, human coronavirus NL63 (HCoV-NL63) and 229E (HCoV-229E) belong to the alphacoronavirus genus, a member of the Coronaviridae family. Coronaviruses are positive-strand RNA viruses with the largest genome of approximately $27-31 \mathrm{~kb}$ in size. ${ }^{4}$ In previous studies, analysis of the spike $(S)$ glycoprotein, nucleocapsid $(N)$, and $1 a$ genes of HCoV-NL63 and HCoV-229E revealed evidence of genetic recombination, genetic drift, and positive selection events as part of the evolution of the virus., Phylogenetically, HCoV-NL63 and HCoV-229E are more closely related to each other than to any other human coronavirus. ${ }^{7}$

HCoV-NL63 and HCoV-229E account for about 5\% of all acute URTI, ${ }^{7-9}$ and in some cases, a small proportion of infections are associated with hospital admission. ${ }^{10,11}$ URTI symptoms such as cough and sore throat are often observed in patients infected with either HCoV-NL63 or HCoV-229E. ${ }^{12,13}$ The prevalence of HCoV-NL63 varies from one study to another; however, in most temperate and tropical countries, it appears to peak around September-April, whereas HCoV-229E is usually detected at low rates throughout the year. ${ }^{14-16}$ In spite of the clinical importance of $\mathrm{HCoV}$ infections, ${ }^{17}$ the prevalence, seasonality, clinical, and phylogenetic characteristics of $\mathrm{HCoVs}$ remain mostly unreported from the tropical region of southeast Asia. On the basis of the $S, N$, and $1 a$ genes of the HCoV-NL63 and HCoV-229E sequences from

*Address correspondence to Kok Keng Tee, Department of Medical Microbiology, Faculty of Medicine, University of Malaya, 50603 Kuala Lumpur, Malaysia. E-mail: k2tee@um.edu.my
Malaysia and also worldwide, we describe the genetic history and phylodynamic profiles of both human alphacoronaviruses using a set of phylogenetic tools.

\section{MATERIALS AND METHODS}

Ethics statement. The study was approved by the University of Malaya Medical Ethics Committee (MEC890.1). Standard, multilingual consent forms permitted by the Medical Ethics Committee were used. Written consent was obtained from all study participants.

Clinical specimens. A total of 2,060 consenting outpatients who presented with acute URTI symptoms were recruited at the Primary Care Clinic of University Malaya Medical Center in Kuala Lumpur, Malaysia, between March 2012 and February 2013. Demographic data such as age, gender, and ethnicity were acquired before the collection of nasopharyngeal swabs. The severity of the URTI symptoms (sneezing, nasal discharge, nasal congestion, headache, sore throat, voice hoarseness, muscle ache, and cough) was graded according to criteria described earlier. ${ }^{18-21}$ The nasopharyngeal swabs were transferred to the laboratory in universal transport media and stored at $-80^{\circ} \mathrm{C}$.

Molecular detection of HCoV-NL63 and HCoV-229E. Extraction of total nucleic acids from the nasopharyngeal swabs was carried out using the magnetic bead-based protocols applied in the NucliSENS easyMAG automated nucleic acid extraction system (BioMérieux, Marcy I'Etoile, France). ${ }^{22,23}$ The presence of respiratory viruses in specimens was examined using the xTAG Respiratory Virus Panel FAST multiplex reverse transcriptase polymerase chain reaction (RT-PCR) assay (Luminex Molecular Diagnostics, Toronto, Canada), which can identify HCoV-NL63, HCoV-229E, HCoV-OC43, $\mathrm{HCoV}-\mathrm{HKU} 1$, and other respiratory viruses and subtypes. ${ }^{24}$ 
Genetic analysis of HCoV-NL63 and HCoV-229E. Gene fragment sequencing of the $S$ (S1 domain), complete $N$, and partial $1 a$ (nsp3) genes was performed for HCoV-NL63 and $\mathrm{HCoV}-229 \mathrm{E}$ specimens. The $\mathrm{S} 1$ is a highly variable receptorbinding domain, whereas the $\mathrm{N}$ and nsp3 are conserved regions within the coronavirus genome, and these three regions are therefore efficiently used for genotyping. ${ }^{5,6}$ Viral RNA was reverse transcribed into complementary DNA (cDNA) using the SuperScript III kit (Invitrogen, Carlsbad, CA) with random hexamers (Applied Biosystems, Carlsbad, CA). The partial $S$ gene (S1 domain) (HCoV-NL63: 1,383 nt [20,413-21,796] and HCoV-229E: 855 nt [20,819-21,674]), complete $N$ gene (HCoV-NL63: 1,133 nt [26,133-27,266] and HCoV-229E: 1,330 nt [25,673-27,003]), and partial 1a (nsp3) gene (HCoV-NL63: 781 nt [5,811-6,592] and HCoV-229E: 766 nt $[5,898-6,664])$ were amplified through PCR using $10 \mu \mathrm{M}$ of newly designed or previously published primers listed in Table 1 . The PCR mixture $(25 \mu \mathrm{L})$ contained cDNA, PCR buffer (10 mM Tris-HCl [pH 8.3], $50 \mathrm{mM} \mathrm{KCl,} 3 \mathrm{mM} \mathrm{MgCl}$, and $0.01 \%$ gelatin), $100 \mu \mathrm{M}$ (each) deoxynucleoside triphosphates, Hi-Spec additive and $4 \mathrm{U} / \mu \mathrm{L}$ BIO-X-ACT Short DNA polymerase (BioLine, London, United Kingdom). The cycling conditions were as follows: initial denaturation at $95^{\circ} \mathrm{C}$ for 5 minutes followed by 40 cycles of $94^{\circ} \mathrm{C}$ for 1 minute, $54.5^{\circ} \mathrm{C}$ for 1 minute, $72^{\circ} \mathrm{C}$ for 1 minute, and a final extension at $72^{\circ} \mathrm{C}$ for 10 minutes. PCR reactions were performed in a C1000 Touch automated thermal cycler (Bio-Rad, Hercules, CA). Nested/semi-nested PCR was performed if necessary, under the same cycling conditions at 30 cycles. Purified PCR products were sequenced using the ABI PRISM 3730XL DNA Analyzer (Applied Biosystems). The nucleotide sequences were codon aligned with relevant complete and partial $\mathrm{HCoV}$ NL63 and HCoV-229E reference sequences retrieved from the GenBank. $5,6,28-31$

Maximum clade credibility (MCC) trees for the partial $S$ (S1 domain), complete $N$, and partial $1 a$ (nsp3) genes were reconstructed in BEAST (version 1.7). ${ }^{32}$ MCC trees were produced using a relaxed molecular clock, assuming uncorrelated lognormal distribution under the general time-reversible nucleotide substitution model with a proportion of invariant sites $(\mathrm{GTR}+\mathrm{I})$ and a constant coalescent/exponential tree model. The Markov chain Monte Carlo run was set at $6 \times 10^{6}$ steps long sampled every 10,000 state. The trees were annotated using Tree Annotator program included in the BEAST package, after a $10 \%$ burn-in, and visualized in FigTree (version 1.3.1). ${ }^{33}$ The evolutionary history and divergence time (in calendar year) for the HCoV-NL63 and HCoV-229E genotypes were also assessed. The mean divergence time and the $95 \%$ highest posterior density regions were evaluated. The bestfitting model was determined by the Bayes factor using marginal likelihood analysis implemented in Tracer (version 1.5). ${ }^{32}$ The substitution rate of $3.3 \times 10^{-4}$ substitutions/site/year for the $S$ gene of human alphacoronavirus estimated previously was used for the divergence time inference. ${ }^{5}$

Maximum likelihood (ML) phylogenetic trees were also reconstructed for the three regions in the phylogenetic analysis using parsimony (PAUP 4.0) software $^{34}$ with a HasegawaKishino-Yano nucleotide substitution model plus discrete gamma categories. The statistical robustness and reliability of the branching orders were evaluated by a bootstrap analysis of 1,000 replicates. To investigate the genetic relatedness among the HCoV-NL63 and HCoV-229E genotypes, inter-genotype pairwise nucleotide distances were estimated for the $S$ gene using MEGA 5.1. ${ }^{35}$ Such analysis was not implemented for the $N$ and $1 a$ genes due to their high genetic invariability across HCoV-NL63 and HCoV-229E genotypes. ${ }^{5,6}$

Statistical analysis. All categorical variables were analyzed using the two-tailed Fisher's exact test $/ \chi^{2}$ test by the Statistical Package for the Social Sciences (release 16.0; IBM Corp., Chicago, IL). $P$ values $<0.05$ were considered significant.

Nucleotide sequences. HCoV-NL63 and HCoV-229E nucleotide sequences produced in the study have been deposited in GenBank under the accession nos. KT359730-KT359913.

\section{RESULTS AND DISCUSSION}

Detection of HCoV-NL63 and HCoV-229E in nasopharyngeal swabs. In the current cross-sectional study, a total of

TABLE 1

Polymerase chain reaction primers for HCoV-NL63 and HCoV-229E

\begin{tabular}{|c|c|c|c|c|c|}
\hline Target gene & $\mathrm{HCoV}$ & Primer & Location* & Sequence $\left(5^{\prime}-3^{\prime}\right)$ & Reference \\
\hline \multirow[t]{8}{*}{ Spike $(S)$} & \multirow[t]{4}{*}{ NL63 } & SP1F & $20,390-20,412$ & Forward: TGAGTTTGATTAAGAGTGGTAGG & 25 \\
\hline & & SP2F & $20,397-20,418$ & Forward (nested): GATTAAGAGTGGTAGGTTGTTG & 25 \\
\hline & & SP1R & $21,809-21,828$ & Reverse: CAAACTGCAAGTGCTCACAC & 25 \\
\hline & & SP2R & $21,797-21,816$ & Reverse (nested): GCTCACACTGCAACTTTTCA & 25 \\
\hline & \multirow[t]{4}{*}{$229 \mathrm{E}$} & LPS1 & $20,732-20,751$ & Forward: AATAATTGGTTCCTTCTAAC & 26 \\
\hline & & JH1 & $20,797-20,818$ & Forward (nested): TTTGTTGCTTAATTGCTTATGG & 26 \\
\hline & & LPR & $21,710-21,728$ & Reverse: AACATACACTGCCAAATTT & This study \\
\hline & & $\mathrm{JH} 2$ & $21,675-21,694$ & Reverse (nested): TTTGCCAAAAGAAAAAGGGC & \\
\hline \multirow[t]{6}{*}{ Nucleocapsid $(N)$} & \multirow[t]{2}{*}{$\begin{array}{l}\text { NL63 } \\
\quad \text { and 229E }\end{array}$} & $\alpha \mathrm{N}-\mathrm{F}$ & $\begin{array}{l}26,102-26,127 \text { (NL63) } \\
25,652-25,672 \text { (229E) }\end{array}$ & Forward: ARRTTGCTTCATTTWWTCTAA & This study \\
\hline & & $\alpha \mathrm{N}-\mathrm{Fn}$ & $26,112-26,132$ & Forward (nested): ATTTWWTCTAAACTAAACRAA & This study \\
\hline & \multirow[t]{2}{*}{ NL63 } & NL-NR & $27,278-27,299$ & Reverse: ATAATAAACAKTCAACTGGAAT & This study \\
\hline & & NL-NRn & $27,267-27,287$ & Reverse (nested): CAACTGGAATTACAAAACAAT & This study \\
\hline & \multirow[t]{2}{*}{$229 \mathrm{E}$} & E-NR & $27,046-27,063$ & Reverse: GATCCTTGTCAAGCCAAA & This study \\
\hline & & E-NRn & $26,882-26,900$ & Reverse (nested): AAAATTCCAACTAAAGCCT & This study \\
\hline \multirow[t]{6}{*}{$1 a$} & \multirow[t]{3}{*}{ NL63 } & SS5852-5Pf & $5,778-5,798$ & Forward: CTTTTGATAACGGTCACTATG & \\
\hline & & P3E2-5Pf & $5,789-5,810$ & Forward (semi-nested): GGTCACTATGTAGTTTATGATG & 27 \\
\hline & & NL-1aR & $6,593-6,616$ & Reverse: CTCATTACATAAAACATCRAACGG & This study \\
\hline & \multirow[t]{3}{*}{$229 \mathrm{E}$} & $\mathrm{E}-1 \mathrm{aF}$ & $5,865-5,585$ & Forward: CTGTTGAYAAAGGTCATTATA & This study \\
\hline & & E-1aFn & $5,876-5,897$ & Forward (semi-nested): GGTCATTATACTGTTTATGAYA & This study \\
\hline & & E-1aR & $6,665-6,688$ & Reverse: TTCATCACAAATAACATCAAATGG & This study \\
\hline
\end{tabular}

* Nucleotide location was determined based on the HCoV-NL63 (NC_005831) and HCoV-229E (NC_002645) reference sequences. 
TABLE 2

Demographic data of 68 adult outpatients infected with human alphacoronavirus in Kuala Lumpur, Malaysia, 2012-2013

\begin{tabular}{lcrr}
\hline \multicolumn{1}{c}{ Factor } & HCoV-NL63 $(N=45)$ & HCoV-229E $(N=23)$ & $P$ value \\
\hline Gender & & & 0.80 \\
$\quad$ Male & $25(55.6 \%)$ & $12(52.2 \%)$ & \\
Female & $20(44.4 \%)$ & $11(47.8 \%)$ & \\
Age & $13(28.9 \%)$ & $7(30.4 \%)$ & 0.45 \\
$\quad<40$ & $10(22.2 \%)$ & $8(34.8 \%)$ & \\
$40-60$ & $22(48.9 \%)$ & $8(34.8 \%)$ & \\
$>60$ & & & 0.99 \\
Symptoms & $42(93.3 \%)$ & $20(87.0 \%)$ & \\
$\quad$ Sneezing & $38(84.4 \%)$ & $19(82.6 \%)$ & \\
Nasal discharge & $29(64.4 \%)$ & $15(65.2 \%)$ & \\
Nasal congestion & $23(51.1 \%)$ & $13(56.5 \%)$ & \\
Headache & $32(68.9 \%)$ & $14(60.9 \%)$ & \\
Sore throat & $35(77.8 \%)$ & $15(65.2 \%)$ & \\
Hoarseness of voice & $27(60.0 \%)$ & $16(69.6 \%)$ & \\
Muscle ache & $43(95.6 \%)$ & $20(87.0 \%)$ & \\
$\quad$ Cough & $11(24.5 \%)$ & $5(21.8 \%)$ & 0.08 \\
Ethnicity & $24(53.3 \%)$ & $7(30.4 \%)$ & \\
$\quad$ Malay & $10(22.2 \%)$ & $11(47.8 \%)$ & \\
Chinese & & & \\
Indian & & & \\
\hline
\end{tabular}

2,060 nasopharyngeal swab specimens collected from Kuala Lumpur, Malaysia, throughout a 12-month study period (March 2012 to February 2013), were screened for the presence of HCoV-NL63 and HCoV-229E using the multiplex RT-PCR method, as an alternative approach to other detection methods such as cell culture. ${ }^{36}$ Human alphacoronavirus was identified in 68 (3.3\%) subjects; HCoV-NL63 and HCoV-229E were detected in 45/2,060 (2.2\%) and 23/2,060 (1.1\%) patients, respectively. These findings are consistent with the global average prevalence of human alphacoronavirus, which ranges between $1 \%$ and $10 \%$, with $\mathrm{HCoV}-229 \mathrm{E}$ generally detected at lower rates than HCoV-NL63. ${ }^{8-10,27,37-40}$ In contrast to an earlier study, ${ }^{41}$ no coinfection of alphaand betacoronavirus (HCoV-OC43 and HCoV-HKU1) was observed within an individual. Age, gender, and ethnicity of the patients were summarized in Table 2. A peak in the number of HCoV-NL63 infections was recorded for the period between June and October 2012, although the number of patients with URTI symptoms screened during those months was relatively low (Figure 1). This pattern of virus prevalence corroborates with that observed in neighboring country Thailand, in which a peak of HCoV-NL63 incidence was recorded in September. ${ }^{14}$ In contrast, studies from temperate regions commonly reported a higher prevalence of $\mathrm{HCoV}$ NL63 during winter seasons. ${ }^{7-9,42}$ However, the number of HCoV-229E infections detected in Malaysia was low, with no significant peak observed throughout the year, similar to other studies reported worldwide. ${ }^{14,38,43}$ It is important to note that the study was performed in a relatively short duration, therefore limiting the epidemiological and disease trend comparison with reports from other countries.

Phylogenetic analysis of the $\boldsymbol{S}, \boldsymbol{N}$, and $1 \mathrm{a}$ genes. A total of $42 / 45(93.3 \%)$ partial $S$ (S1 domain) and 43/45 (95.6\%) of each complete $N$ and partial $1 a$ (nsp3) genes were successfully sequenced from HCoV-NL63 specimens. Amplification of these genes was difficult for two xTAG-positive HCoVNL63 specimens, possibly due to their low viral copy number. Phylogenetic analysis of HCoV-NL63 (Figure 2 and Supplemental Figure 1) showed that 27 subjects (27/43, 62.8\%) in the study belonged to genotype B (supported by a posterior probability of 1.0 and bootstrap value of $100 \%$ at the internal nodes of the MCC and ML trees of the $S$ gene, respectively, with an intra-group pairwise genetic distance of $0.6 \% \pm 0.1 \%$ ) together with previously reported sequences from the United States, Europe, and Asia. 5,25,28,29 Another 16 subjects $(16 / 43,37.2 \%)$ were found to be grouped under genotype $\mathrm{C}$ (supported by a posterior probability of 1.0 and bootstrap value of $67 \%$ at the internal nodes of the MCC and ML trees of the $S$ gene, respectively, with an intra-group pairwise genetic distance of $0.2 \% \pm 0.1 \%$ ) with recently described global sequences. ${ }^{25,28,29}$ Discordance in phylogenetic clustering among the $S, N$, and $1 a$ genes of the $\mathrm{HCoV}$ NL63 Malaysian sequences had been observed (Supplemental Figure 1). On the basis of the $S$ (S1domain) gene analysis, 26 Malaysian strains $(26 / 42 ; 61.9 \%)$ belong to genotype B while another 16 Malaysian strains $(16 / 42 ; 38.1 \%)$ were classified within genotype $\mathrm{C}$. In contrast, sequences of the three HCoV-NL63 genotypes (A, B, and C) appear to be intermingled in the $N$ and $1 a$ phylogenetic trees. Such discordance was similarly reported in earlier studies where it was

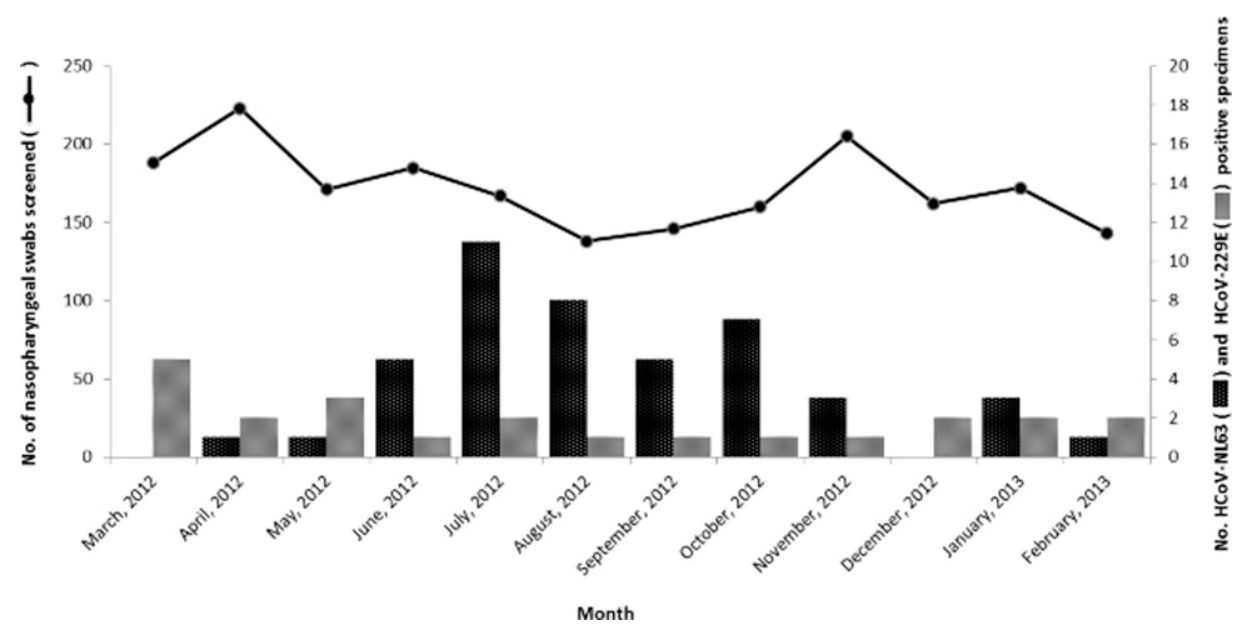

FIGURE 1. Annual distribution of HCoV-NL63 and HCoV-229E among adults with acute upper respiratory tract infections in Kuala Lumpur, Malaysia. The total number of nasopharyngeal swabs screened and the monthly distribution of HCoV-NL63 and HCoV-229E between March 2012 and February 2013 were presented. 


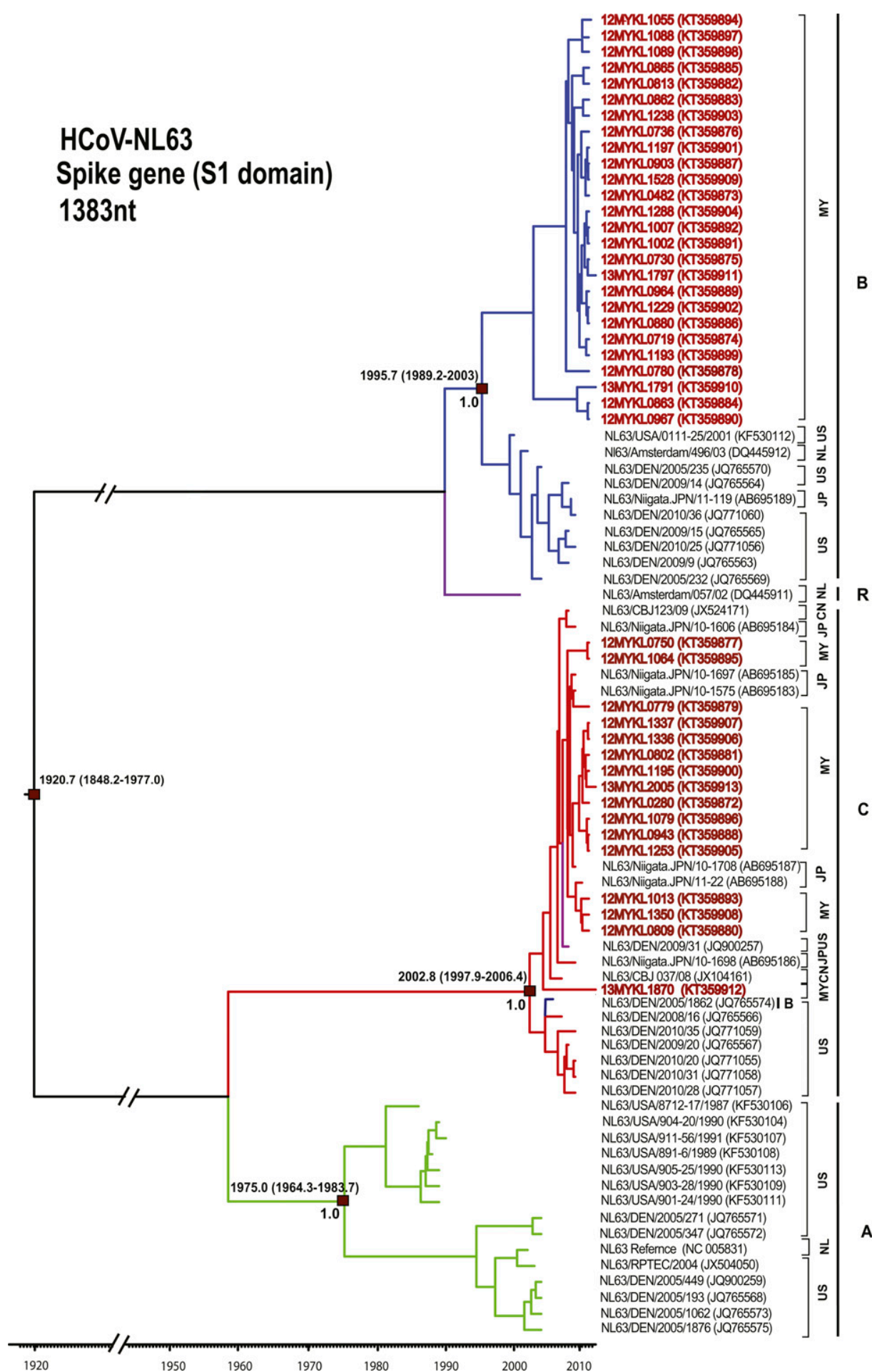

FIGURE 2. Maximum clade credibility tree of HCoV-NL63. Spike gene (S1 domain) sequences (1,383 nucleotides) were analyzed under the relaxed molecular clock with a GTR+I substitution model and a constant size coalescent model implemented in BEAST. Posterior probability values and the estimation of the time of the most recent common ancestors with $95 \%$ highest posterior density were indicated on major nodes. The HCoV-NL63 sequences obtained in this study were color coded and HCoV-NL63 genotypes A-C were indicated; green = genotype A, blue = genotype $\mathrm{B}$, and red = genotype $\mathrm{C}$. The recombinant genotype is indicated by purple color. The sampling site for each sequence was indicated by codes for the representation of countries. Country codes are as follows; MY = Malaysia; US = United States; JP = Japan; NL = Netherlands; $\mathrm{CN}=$ China

confirmed that such phylogenetic pattern was resulted from multiple recombination events along the HCoV-NL63 genome, in addition to the fact that the $\mathrm{S} 1$ region sequenced in this study is considered the most variable along the genome, while the $N$ and $1 a$ (nsp3) genes are too conserved. ${ }^{5}$ To estimate the genetic diversity between HCoV-NL63 genotypes A, B, and C, inter-genotype pairwise genetic distance was assessed for the $S$ gene (Table 3). Genetic distances between genotypes A versus B 
TABLE 3

The genetic diversity among alphacoronavirus genotypes in the spike gene

\begin{tabular}{lccccc}
\hline & HCoV & \multicolumn{4}{c}{ Genetic distance } \\
\hline NL63 & & genotype A & genotype B & genotype C \\
& genotype A & - & $0.8 \dagger$ & 0.5 & \\
& genotype B & $7.6^{*}$ & - & 0.6 & \\
$229 \mathrm{E}$ & genotype C & 2.1 & 6.7 & - & \\
& & group 1 & group 2 & group 3 & group 4 \\
& group 1 & - & 0.4 & 0.6 & 0.7 \\
& group 2 & 1.5 & - & 0.3 & 0.4 \\
& group 3 & 2.5 & 1.2 & - & 0.3 \\
& group 4 & 3.5 & 2.6 & 1.5 & - \\
\hline *Pairwise genetic distances are expressed in percentage (\%) of nucleotide difference.
\end{tabular}

$\dagger$ Standard error estimates of the mean genetic distances are shown in the upper diagonal.

and $\mathrm{B}$ versus $\mathrm{C}$ were high (more than $5.0 \%$ ), compared with that between genotypes $\mathrm{A}$ versus $\mathrm{C}$, which was at $2.1 \%$. This is consistent with the phylogenetic tree topology in which genotypes $\mathrm{A}$ and $\mathrm{C}$ were more closely related and probably shared a common ancestor.

At least one gene $(S, N$, and/or $1 a)$ was successfully sequenced from 23 positively tested $\mathrm{HCoV}-229 \mathrm{E}$ specimens $(16,18$, and 22 of HCoV-229E $S, N$, and $1 a$ genes, respectively). Phylogenetic analysis revealed that all of the HCoV$229 \mathrm{E}$ sequences obtained in this study were classified with group 4, which includes isolates that have been globally circulating since 2001 (Figure 3 and Supplemental Figure 2). 6,30,31 The group was supported by a posterior probability of 1.0 and bootstrap value of $100 \%$ at the internal nodes of the MCC and ML trees of the $S$ gene, respectively, with an intra-group pairwise genetic distance of $0.3 \% \pm 0.1 \%$. Such phylogenetic data were comparable to those obtained from the $N$ tree, resulted from the hot substitution spots in the $\mathrm{S} 1$ and $\mathrm{N}$ regions of the HCoV-229E genome. ${ }^{30}$ The four HCoV-229E groups could not be clearly defined within the $1 a$ gene tree because of the limited number of reference sequences available in the public database (Supplemental Figure 2). Intergenotype pairwise genetic distance was generally low (below $5.0 \%$ ) in the $S$ gene among groups $1-4$ (Table 3 ).

Estimation of divergence times. The molecular clock analysis of HCoV-NL63 and HCoV-229E was performed using the coalescent-based Bayesian relaxed molecular clock under the constant and exponential tree models (Figures 2 and 3). The mean evolutionary rates for the $S$ gene of HCoV-NL63 and $\mathrm{HCoV}-229 \mathrm{E}$ were newly estimated based on the constant tree model at $4.3 \times 10^{-4}\left(2.3-6.7 \times 10^{-4}\right)$ and $3.9 \times 10^{-4}$ $\left(1.3-6.4 \times 10^{-4}\right)$ substitutions/site/year, respectively. These results were similar to the previously reported substitution rate of the alphacoronavirus $S$ gene $\left(3.3 \times 10^{-4}\right.$ substitutions/ site/year). ${ }^{5}$ The evolutionary analysis indicated that the time of the most recent common ancestor (tMRCA) of $\mathrm{HCoV}$ NL63 was dated back to the 1920s, while the estimated divergence time of genotype A was dated to 1975, followed by genotype B around 1996 and genotype C in 2003 (Figure 2). Furthermore, the divergence time of HCoV-229E

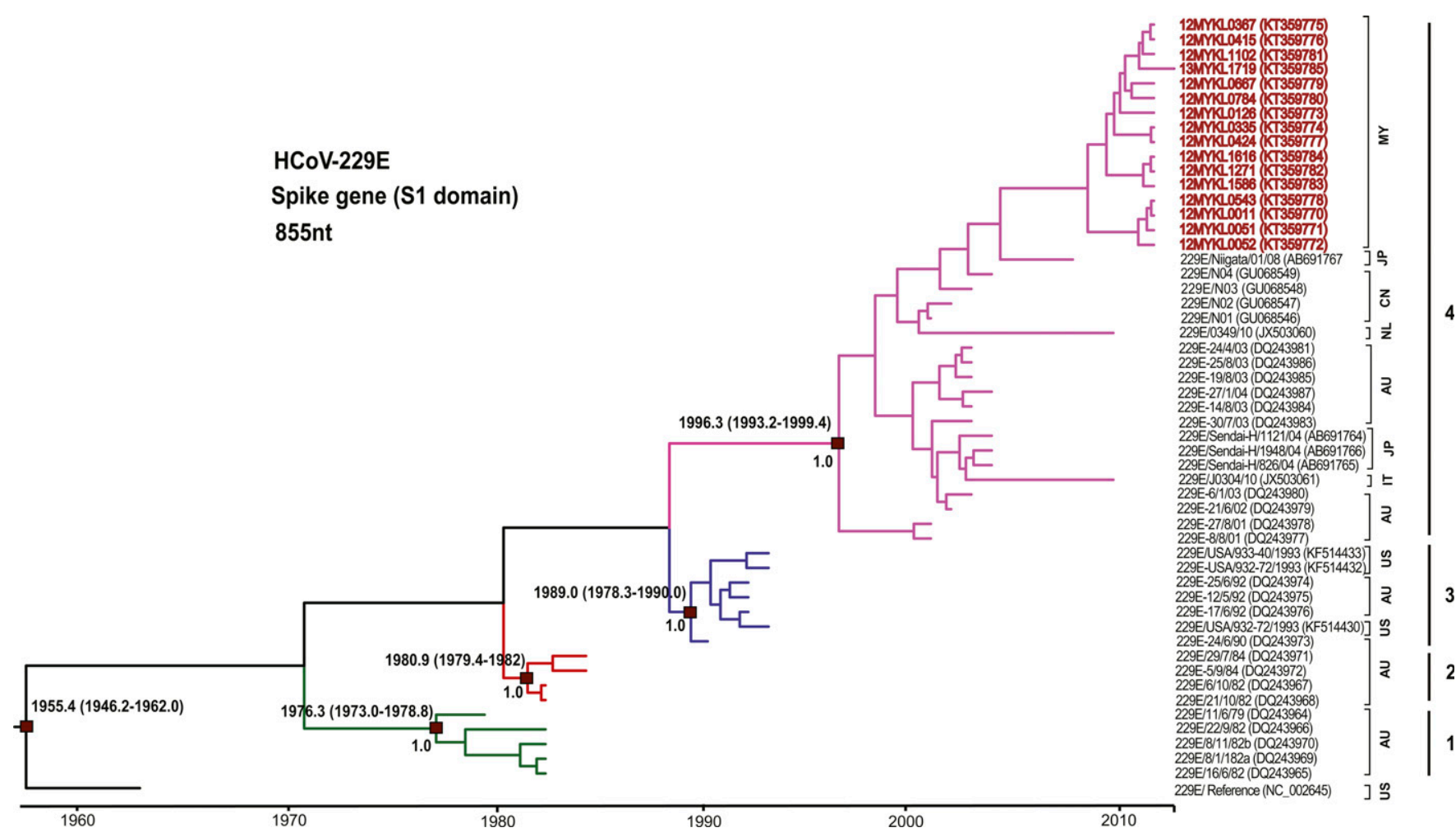

FIGURE 3. Maximum clade credibility tree of HCoV-229E. Spike gene ( $\mathrm{S} 1$ domain) sequences (855 nucleotides) were analyzed under the relaxed molecular clock with a GTR $+\mathrm{I}$ substitution model and a constant size coalescent model implemented in BEAST. Posterior probability values and the estimation of the time to the most recent common ancestors with $95 \%$ highest posterior density were indicated on the major nodes. The HCoV-229E sequences obtained in this study were color coded and the HCoV-229E groups 1-4 were indicated green = genotype 1, red $=$ genotype 2 , blue $=$ genotype 3 , and purple $=$ genotype 4 . The sampling site for each sequence was indicated by codes for the representation of countries. Country codes are as follows; MY = Malaysia; US = United States; JP = Japan; NL = Netherlands; CN = China; AU = Australia; IT = Italy. 
(Figure 3) was estimated around 1955 while the tMRCA of group 1 diverged in 1976, followed by that of group 2 in 1981, group 3 in 1989, and group 4 in 1996. The appearance of groups 1-4 in a timely ordered manner would give strength to the earlier reported hypothesis that positive selection and genetic drift play a major role in the evolution of $\mathrm{HCoV}$ $229 \mathrm{E}$. $^{6,30}$ To the best of our knowledge, this is the first study that reported the divergence times of human alphacoronavirus genotypes. In addition, the most recently reported $\mathrm{HCoV}$ 229E strains (between 2001 and 2013) from major parts of the world belong to group 4. In accordance with earlier studies, genotype replacement is evident within $\mathrm{HCoV}-229 \mathrm{E}$, although sampling bias may also influence the results. ${ }^{6,30}$ Bayes factor analysis showed insignificant differences (Bayes factor less than 3.0) between the constant and exponential coalescent models of demographic analysis, in which the divergence times estimated using the constant coalescent tree model were similar to those calculated using the exponential model (Supplemental Table 1).

Clinical symptoms assessment. Clinical findings of the URTI symptoms (sneezing, nasal discharge, nasal congestion, headache, sore throat, hoarseness of voice, muscle ache, and cough) and their severity levels (none, moderate, and severe) were analyzed using the two-tailed Fisher's exact test. The association between symptom severity and $\mathrm{HCoV}-\mathrm{NL} 63 / \mathrm{HCoV}$ $229 \mathrm{E}$ infection was insignificant $(P$ values $>0.05)$ (Supplemental Table 2). In line with previous clinical studies, ${ }^{10,44,45}$ the majority of patients infected with HCoV-NL63 and HCoV$229 \mathrm{E}$ presented with at least one respiratory symptom that was moderately severe.

In summary, this study provides insight into the phylogeny and evolution of the HCoV-NL63 and HCoV-2293E genotypes. Genetic characterization of human alphacoronavirus isolates currently circulating in Malaysia indicates the circulation of globally prevalent genotypes in the tropical region of southeast Asia. This study has detailed the genetic history of HCoV-NL63 and HCoV-229E genotypes. Since alphacoronavirus evolve through recombination, positive selection, and genetic drift events, continuous molecular surveillance of human alphacoronavirus is warranted to keep track on the evolution of the virus in southeast Asia.

Received November 12, 2015. Accepted for publication January 13, 2016.

Published online February 26, 2016.

Note: Supplemental tables and figures appear at www.ajtmh.org.

Acknowledgments: We would like to thank Nyoke Pin Wong, Nur Ezreen Syafina, Farhat A. Avin, Chor Yau Ooi, Sujarita Ramanujam, Nirmala K. Sambandam, Nagammai Thiagarajan, and See Wie Teoh for assistance and support.

Financial support: This work was supported by grants from the Ministry of Education, Malaysia: High Impact Research UM.C/625/ 1/HIR/MOE/CHAN/02/02 to Kok Keng Tee.

Disclaimer: The outcomes and conclusions that this study has reported were drawn by the authors and do not necessarily represent the views or policies of the institutions of affiliation.

Authors' addresses: Maryam Nabiel Al-Khannaq, Kim Tien Ng, Xiang Yong Oong, Yong Kek Pang, and Adeeba Kamarulzaman, Department of Medicine, Faculty of Medicine, University of Malaya, Kuala Lumpur, Malaysia, E-mails: maryasala@gmail.com,kim_honcho@yahoo.com, oxy123@siswa.um.edu.my, ykpang@ummc.edu.my, and adeeba@ummc .edu.my. Yutaka Takebe, Department of Medicine, Faculty of Medicine, University of Malaya, Kuala Lumpur, Malaysia, AIDS Research
Center, National Institute of Infectious Diseases, Toyama, Shinjuku-ku, Tokyo, Japan, and School of Medicine, Yokohama City University, Yokohama, Kanagawa, Japan, E-mail: takebe@niid.go.jp. Jack Bee Chook, Department of Medicine, Faculty of Medicine, University of Malaya, Kuala Lumpur, Malaysia, and Department of Health Sciences, Faculty of Health and Life Sciences, Management and Science University, Selangor, Malaysia, E-mail: jackbee2002@um.edu.my. Nik Sherina Hanafi, Department of Primary Care Medicine, Faculty of Medicine, University of Malaya, Kuala Lumpur, Malaysia, E-mail: sherina@ ummc.edu.my. Kok Keng Tee, Department of Medical Microbiology, Faculty of Medicine, University of Malaya, Kuala Lumpur, Malaysia, E-mail: k2tee@um.edu.my.

\section{REFERENCES}

1. Tyrrell DAJ, Bynoe ML, 1965. Cultivation of a novel type of common-cold virus in organ cultures. Br Med J 5448: 1467-1470.

2. McIntosh K, Becker WB, Chanock RM, 1967. Growth in sucklingmouse brain of "IBV-like" viruses from patients with upper respiratory tract disease. Proc Natl Acad Sci USA 58: 2268-2273.

3. Hendley J, Fishburne H, Gwaltney J Jr, 1972. Coronavirus infections in working adults. Eight-year study with $229 \mathrm{E}$ and OC 43. Am Rev Respir Dis 105: 805-811.

4. Lai M, Perlman S, Anderson J, 2006. Coronaviridae. Knipe D, Howley PM, eds. Fields Virology, 5th edition. Philadelphia, PA: Lippincott Williams and Wilkins, 1305-1335.

5. Pyrc K, Dijkman R, Deng L, Jebbink MF, Ross HA, Berkhout B, van der Hoek L, 2006. Mosaic structure of human coronavirus NL63, one thousand years of evolution. J Mol Biol 364: 964-973.

6. Chibo D, Birch C, 2006. Analysis of human coronavirus 229E spike and nucleoprotein genes demonstrates genetic drift between chronologically distinct strains. J Gen Virol 87: 1203-1208.

7. Dijkman R, van der Hoek L, 2009. Human coronaviruses $229 \mathrm{E}$ and NL63: close yet still so far. J Formos Med Assoc 108: 270-279.

8. Gerna G, Percivalle E, Sarasini A, Campanini G, Piralla A, Rovida F, Genini E, Marchi A, Baldanti F, 2007. Human respiratory coronavirus HKU1 versus other coronavirus infections in Italian hospitalised patients. J Clin Virol 38: 244-250.

9. Vabret A, Dina J, Gouarin S, Petitjean J, Tripey V, Brouard J, Freymuth F, 2008. Human (non-severe acute respiratory syndrome) coronavirus infections in hospitalised children in France. J Paediatr Child Health 44: 176-181.

10. Bastien N, Anderson K, Hart L, Van Caeseele P, Brandt K, Milley D, Hatchette T, Weiss EC, Li Y, 2005. Human coronavirus NL63 infection in Canada. $J$ Infect Dis 191: 503-506.

11. van Elden LJ, van Loon AM, van Alphen F, Hendriksen KA, Hoepelman AI, van Kraaij MG, Oosterheert JJ, Schipper P, Schuurman R, Nijhuis M, 2004. Frequent detection of human coronaviruses in clinical specimens from patients with respiratory tract infection by use of a novel real-time reversetranscriptasepolymerase chain reaction. J Infect Dis 189: $652-657$.

12. Kapikian AZ, James HD Jr, Kelly SJ, Dees JH, Turner HC, McIntosh K, Kim HW, Parrott RH, Vincent MM, Chanock $\mathrm{RM}, 1969$. Isolation from man of "avian infectious bronchitis virus-like" viruses (coronaviruses) similar to $229 \mathrm{E}$ virus, with some epidemiological observations. J Infect Dis 119: 282-290.

13. van der Hoek L, 2007. Human coronaviruses: what do they cause? Antivir Ther 12: 651-658.

14. Dare RK, Fry AM, Chittaganpitch M, Sawanpanyalert P, Olsen SJ, Erdman DD, 2007. Human coronavirus infections in rural Thailand: a comprehensive study using real-time reversetranscription polymerase chain reaction assays. J Infect Dis 196: 1321-1328.

15. Gaunt ER, Hardie A, Claas E, Simmonds P, Templeton KE, 2010. Epidemiology and clinical presentations of the four human coronaviruses 229E, HKU1, NL63, and OC43 detected over 3 years using a novel multiplex real-time PCR method. J Clin Microbiol 48: 2940-2947.

16. Leung TF, Li CY, Lam WY, Wong GW, Cheuk E, Ip M, Ng PC, Chan PK, 2009. Epidemiology and clinical presentations of human coronavirus NL63 infections in Hong Kong children. J Clin Microbiol 47: 3486-3492. 
17. Garbino J, Crespo S, Aubert JD, Rochat T, Ninet B, Deffernez C, Wunderli W, Pache JC, Soccal PM, Kaiser L, 2006. A prospective hospital-based study of the clinical impact of nonsevere acute respiratory syndrome (non-SARS)-related human coronavirus infection. Clin Infect Dis 43: 1009-1015.

18. Jackson GG, Dowling HF, Spiesman IG, Boand AV, 1958. Transmission of the common cold to volunteers under controlled conditions. I. The common cold as a clinical entity. AMA Arch Intern Med 101: 267-278.

19. Turner RB, Wecker MT, Pohl G, Witek TJ, McNally E, St George R, Winther B, Hayden FG, 1999. Efficacy of tremacamra, a soluble intercellular adhesion molecule 1 , for experimental rhinovirus infection: a randomized clinical trial. JAMA 281: 1797-1804.

20. Yale SH, Liu K, 2004. Echinacea purpurea therapy for the treatment of the common cold: a randomized, doubleblind, placebo-controlled clinical trial. Arch Intern Med 164: 1237-1241.

21. Zitter JN, Mazonson PD, Miller DP, Hulley SB, Balmes JR, 2002. Aircraft cabin air recirculation and symptoms of the common cold. JAMA 288: 483-486.

22. Boom R, Sol C, Salimans M, Jansen C, Wertheim-van Dillen P, van der Noordaa J, 1990. Rapid and simple method for purification of nucleic acids. J Clin Microbiol 28: 495-503.

23. Chan KH, Yam WC, Pang CM, Chan KM, Lam SY, Lo KF, Poon LL, Peiris JM, 2008. Comparison of the NucliSens easyMAG and Qiagen BioRobot 9604 nucleic acid extraction systems for detection of RNA and DNA respiratory viruses in nasopharyngeal aspirate samples. J Clin Microbiol 46: 2195-2199.

24. Jokela P, Piiparinen H, Mannonen L, Auvinen E, Lappalainen M, 2012. Performance of the Luminex xTAG Respiratory Viral Panel Fast in a clinical laboratory setting. J Virol Methods 182: 82-86.

25. Kon M, Watanabe $\mathrm{K}$, Tazawa $\mathrm{T}$, Watanabe $\mathrm{K}$, Tamura $\mathrm{T}$, Tsukagoshi H, Noda M, Kimura H, Mizuta K, 2012. Detection of human coronavirus NL63 and OC43 in children with acute respiratory infections in Niigata, Japan, between 2010 and 2011. Jpn J Infect Dis 65: 270-272.

26. Hays JP, Myint SH, 1998. PCR sequencing of the spike genes of geographically and chronologically distinct human coronaviruses 229E. J Virol Methods 75: 179-193.

27. van der Hoek L, Pyrc K, Jebbink MF, Vermeulen-Oost W, Berkhout RJ, Wolthers KC, Wertheim-van Dillen PM, Kaandorp J, Spaargaren J, Berkhout B, 2004. Identification of a new human coronavirus. Nat Med 10: 368-373.

28. Dominguez SR, Sims GE, Wentworth DE, Halpin RA, Robinson CC, Town CD, Holmes KV, 2012. Genomic analysis of 16 Colorado human NL63 coronaviruses identifies a new genotype, high sequence diversity in the $\mathrm{N}$-terminal domain of the spike gene and evidence of recombination. J Gen Virol 93: 2387-2398.

29. Geng H, Cui L, Xie Z, Lu R, Zhao L, Tan W, 2012. Characterization and complete genome sequence of human coronavirus NL63 isolated in China. $J$ Virol 86: 9546-9547.

30. Farsani SM, Dijkman R, Jebbink MF, Goossens H, Ieven M, Deijs M, Molenkamp R, van der Hoek L, 2012. The first complete genome sequences of clinical isolates of human coronavirus 229E. Virus Genes 45: 433-439.
31. Shirato K, Kawase M, Watanabe O, Hirokawa C, Matsuyama S, Nishimura H, Taguchi F, 2012. Differences in neutralizing antigenicity between laboratory and clinical isolates of HCoV$229 \mathrm{E}$ isolated in Japan in 2004-2008 depend on the S1 region sequence of the spike protein. J Gen Virol 93: 1908-1917.

32. Drummond AJ, Rambaut A, 2007. BEAST: Bayesian evolutionary analysis by sampling trees. BMC Evol Biol 7: 214-219.

33. Rambaut A, 2007. FigTree, A Graphical Viewer of Phylogenetic Trees. Available at: http://tree.bio.ed.ac.uk/software/figtree. Accessed July 1, 2014.

34. Swofford DL, 2003. PAUP*. Phylogenetic Analysis Using Parsimony (* And Other Methods). Version 4. Sunderland, United Kingdom: MA Sinauer Associates.

35. Tamura K, Peterson D, Peterson N, Stecher G, Nei M, Kumar S, 2011. MEGA5: molecular evolutionary genetics analysis using maximum likelihood, evolutionary distance, and maximum parsimony methods. Mol Biol Evol 28: 2731-2739.

36. Schildgen O, Jebbink MF, de Vries M, Pyrc K, Dijkman R, Simon A, Müller A, Kupfer B, van der Hoek L, 2006. Identification of cell lines permissive for human coronavirus NL63. J Virol Methods 138: 207-210.

37. Cabeca TK, Bellei N, 2012. Human coronavirus NL-63 infection in a Brazilian patient suspected of H1N1 2009 influenza infection: description of a fatal case. J Clin Virol 53: 82-84.

38. Gaunt ER, Hardie A, Claas EC, Simmonds P, Templeton KE, 2010. Epidemiology and clinical presentations of the four human coronaviruses 229E, HKU1, NL63, and OC43 detected over 3 years using a novel multiplex real-time PCR method. J Clin Microbiol 48: 2940-2947.

39. Moes E, Vijgen L, Keyaerts E, Zlateva K, Li S, Maes P, Pyrc K, Berkhout B, van der Hoek L, Van Ranst M, 2005. A novel pancoronavirus RT-PCR assay: frequent detection of human coronavirus NL63 in children hospitalized with respiratory tract infections in Belgium. BMC Infect Dis 5: 6.

40. Matoba Y, Abiko C, Ikeda T, Aoki Y, Suzuki Y, Yahagi K, Matsuzaki Y, Itagaki T, Katsushima F, Katsushima Y, Mizuta K, 2014. Detection of the human coronavirus 229E, HKU1, NL63, and OC43 between 2010 and 2013 in Yamagata, Japan. Jpn J Infect Dis 68: 138-141.

41. Vabret A, Mourez T, Dina J, van der Hoek L, Gouarin S, Petitjean J, Brouard J, Freymuth F, 2005. Human coronavirus NL63, France. Emerg Infect Dis 11: 1225-1229.

42. Razuri H, Malecki M, Tinoco Y, Ortiz E, Guezala MC, Romero C, Estela A, Breña P, Morales ML, Reaves EJ, Gomez J, Uyeki TM, Widdowson MA, Azziz-Baumgartner E, Bausch DG, Schildgen V, Schildgen O, Montgomery JM, 2015. Human coronavirus-associated influenza-like illness in the community setting in Peru. Am J Trop Med Hyg 93: 1038-1040.

43. Lau SK, Woo PC, Yip CC, Tse H, Tsoi HW, Cheng VC, Lee P, Tang BS, Cheung CH, Lee RA, So LY, Lau YL, Chan KH, Yuen KY, 2006. Coronavirus HKU1 and other coronavirus infections in Hong Kong. J Clin Microbiol 44: 2063-2071.

44. Abdul-Rasool S, Fielding BC, 2010. Understanding human coronavirus HCoV-NL63. Open Virol J 4: 76-84.

45. Lu R, Zhang L, Tan W, Zhou W, Wang Z, Peng K, Ruan L, 2009. Characterization of human coronavirus 229E infection among patients with respiratory symptom in Beijing, Oct-Dec, 2007. Zhonghua Shi Yan He Lin Chuang Bing Du Xue Za Zhi 23: $367-370$ 